\title{
Asymptotic Linear Programming Lower Bounds for the Energy of Minimizing Riesz and Gauss Configurations
}

\author{
D. P. Hardin, T. J. Michaels, and E.B. Saff*
}

April 17, 2018

\begin{abstract}
Utilizing frameworks developed by Delsarte, Yudin and Levenshtein, we deduce linear programming lower bounds (as $N \rightarrow \infty$ ) for the Riesz energy of $N$-point configurations on the $d$-dimensional unit sphere in the so-called hypersingular case; i.e, for non-integrable Riesz kernels of the form $|x-y|^{-s}$ with $s>d$. As a consequence, we immediately get (thanks to the Poppy-seed bagel theorem) lower estimates for the large $N$ limits of minimal hypersingular Riesz energy on compact $d$-rectifiable sets. Furthermore, for the Gaussian potential $\exp \left(-\alpha|x-y|^{2}\right)$ on $\mathbb{R}^{p}$, we obtain lower bounds for the energy of infinite configurations having a prescribed density.
\end{abstract}

\section{Introduction}

Minimal energy configurations have wide ranging applications in various scientific fields such as cryptography, crystallography, viral morphology, as well as in finite element modeling, radial basis functions, and Quasi-Monte-Carlo methods for graphics applications. For a fixed dimension and cardinality, the use of the Delsarte-Yudin linear programming bounds and Levenshtein $1 / N$-quadrature rules are known to provide bounds on the minimal energy and prove universal optimality of some configurations on the sphere $\mathbb{S}^{d}$ (see for example $[8]$ ). The goal of this paper is to adapt these techniques to provide lower bounds on minimal energy for configurations in two different but related contexts. The first is for the large $N$ limit of Riesz energy of $N$-point configurations on a compact $d$-rectifiable set embedded in $\mathbb{R}^{p}$, while the second is for the Gaussian energy of infinite configurations in $\mathbb{R}^{p}$ having a prescribed density. The latter provides an alternative method for obtaining a main result of Cohn and de Courcy-Ireland 7.

The research of the authors was supported, in part, by National Science Foundation grant DMS-1516400. The research of T. Michaels was completed as part of his Ph.D. dissertation at Vanderbilt University. Research for this article was conducted while two of the authors were in residence at the Institute for Computational and Experimental Research in Mathematics in Providence, RI, during the "Point Configurations in Geometry, Physics and Computer Science" program supported by the National Science Foundation under Grant No. DMS1439786 . 
For our results on Riesz potentials we need the following definitions and notations. We say a set $A \subset \mathbb{R}^{p}$ is d-rectifiable if it is the image of a bounded set in $\mathbb{R}^{d}$ under a Lipschitz mapping. For a $d$-rectifiable, closed set $A$ and a lower semicontinuous, symmetric kernel $K: A \times A \rightarrow(-\infty, \infty]$, the $K$-energy of a configuration $\omega_{N}=\left\{x_{1}, \ldots, x_{N}\right\} \subset A$ of $N$ (not necessarily distinct) points is given by

$$
E_{K}\left(\omega_{N}\right):=\sum_{i \neq j} K\left(x_{i}, x_{j}\right)
$$

A commonly arising problem is to minimize the $K$-energy for a fixed number of points and describe the optimal configurations; i.e., to determine

$$
\mathcal{E}_{K}(A, N):=\inf _{\omega_{N} \subset A} E_{K}\left(\omega_{N}\right) .
$$

For point configurations on compact sets we will primarily focus on the Riesz $s$-kernels

$$
K_{s}(x, y):=|x-y|^{-s} \text { for } s>d=\operatorname{dim}(A) ;
$$

that is, in the hypersingular case, which is intimately related to the best-packing problem. We remark that for such hypersingular kernels, the continuous senergy of $A$

$$
\mathcal{I}_{s}[\mu]:=\int_{A} \int_{A} K_{s}(x, y) d \mu(x) d \mu(y)
$$

is infinite for every probability measure $\mu$ supported on $A$, and so the standard methods of potential theory for obtaining large $N$ limits of minimizing point configurations do not apply.

For brevity we hereafter set

$$
E_{s}\left(\omega_{N}\right):=E_{K_{s}}\left(\omega_{N}\right), \quad \mathcal{E}_{s}(A, N):=\mathcal{E}_{K_{s}}(A, N) .
$$

Furthermore, if $A$ is the unit sphere $\mathbb{S}^{d} \subset \mathbb{R}^{d+1}$ and $K(x, y)$ is a kernel on $\mathbb{S}^{d} \times \mathbb{S}^{d}$ of the form $K(x, y)=h(\langle x, y\rangle)$ for some function $h$ on $[-1,1]$, we write

$$
E_{h}\left(\omega_{N}\right)=E_{K}\left(\omega_{N}\right), \quad \mathcal{E}_{h}\left(\mathbb{S}^{d}, N\right)=\mathcal{E}_{K}\left(\mathbb{S}^{d}, N\right) .
$$

In particular,

$$
K_{s}(x, y)=h_{s}(\langle x, y\rangle):=(2-2\langle x, y\rangle)^{-s} .
$$

For fixed cardinalities $N$ and kernels of the form $K(x, y)=h(\langle x, y\rangle)$, a general framework for obtaining lower bounds for minimal energy configurations on the unit sphere was developed by Yudin [29] based on a method of Delsarte, Goethals, and Seidel 12 for spherical designs. This linear programming technique involves maximizing a certain functional defined over a constrained class of functions $f$ that satisfy $f(t) \leq h(t)$ for $t \in[-1,1]$. Combining Yudin's approach with Levenshtein's work [20, 18 on maximal spherical codes, Boyvalenkov et al [5] derived lower bounds for discrete energy that are 'universal' in the sense that they hold whenever the potential function $h(t)$ is absolutely monotone on $[-1,1)$; that is, when $h^{(k)}(t)$ exists and is non-negative for $t \in[-1,1)$ for all $k \geq 0$, and $h(1):=\lim _{t \rightarrow 1^{-}} h(t)$, which may be $+\infty$.

In the present paper, we use this framework to derive asymptotic lower bounds as $N \rightarrow \infty$ for $\mathcal{E}_{s}\left(\mathbb{S}^{d}, N\right)$ in the case $s>d$. These results for the sphere, 
in turn, have application to the broader class of energy problems on $d$-rectifiable sets. Indeed, this is a consequence of the localized nature of the potentials $h_{s}$ as expressed in the following result, which is known as the Poppy-seed bagel theorem.

Theorem 1.1 ([15], 3]). For any d-rectifiable closed set $A \subset \mathbb{R}^{p}$ and any $s>d$, there exists a positive, finite constant $C_{s, d}$, independent of $A$ such that

$$
\lim _{N \rightarrow \infty} \frac{\mathcal{E}_{s}(A, N)}{N^{1+s / d}}=\frac{C_{s, d}}{\mathcal{H}_{d}(A)^{s / d}}
$$

Furthermore, any sequence of $N$-point s-energy minimizing configurations is asymptotically uniformly distributed with respect to d-dimensional Hausdorff measure restricted to $A$.

In (1), $\mathcal{H}_{d}(A)$ denotes the $d$-dimensional Hausdorff measure of $A$ with the normalization that the $d$-dimensional unit cube embedded in $\mathbb{R}^{p}$ has measure 1 .

In dimension $d=1$, it is known 21] that $C_{s, 1}=2 \zeta(s)$, but for all other dimensions the exact values of $C_{s, d}$ have not as yet been proven. However, the following relation between $C_{s, d}$ and the optimal packing density in $\mathbb{R}^{d}$ was established in 2]:

$$
\lim _{s \rightarrow \infty}\left[C_{s, d}\right]^{1 / s}=\frac{1}{C_{\infty, d}}, \quad C_{\infty, d}:=2\left[\frac{\Delta_{d}}{\mathcal{H}_{d}\left(\mathbb{B}^{d}\right)}\right]^{1 / d}
$$

where $\Delta_{d}$ is the largest sphere packing density in $\mathbb{R}^{d}$. The only dimensions for which $\Delta_{d}$ is known at present are $d=1,2,3$ and, more recently, $d=8$ and $d=24$ (see [26] and [9]). In these special dimensions, $\Delta_{d}$ is attained by lattice packings, which is not expected to be the case for general dimensions.

Clearly, any sequence of configurations on a set $A$ provides an upper bound for $C_{s, d}$. Furthermore, it is straightforward (see, for example, [6], Proposition 1) to establish that

$$
C_{s, d} \leq \min _{\Lambda \subset \mathbb{R}^{d}}|\Lambda|^{s / d} \zeta_{\Lambda}(s),
$$

where the minimum is taken over all lattices $\Lambda \subset \mathbb{R}^{d}$ with covolume $|\Lambda|>0$ and

$$
\zeta_{\Lambda}(s):=\sum_{0 \neq x \in \Lambda}|x|^{-s}
$$

is the Epstein zeta function for the lattice. Regarding equality, the following conjecture is well known [6], [8]:

Conjecture 1.2. For $d=2,4,8$, and 24,

$$
C_{s, d}=\widetilde{C}_{s, d}:=\left|\Lambda_{d}\right|^{\frac{s}{d}} \zeta_{\Lambda_{d}}(s), \quad s>d,
$$

where $\Lambda_{2}$ is the equi-triangular lattice, $\Lambda_{4}$ the $D_{4}$ lattice, $\Lambda_{8}$ the $E_{8}$ lattice, and $\Lambda_{24}$ the Leech lattice.

General lower bounds on $C_{s, d}$ have been less studied. A crude but simple lower bound arises from the following convexity argument (cf. 16]). 
Let $\omega_{N}^{*}=\left\{x_{1}, \ldots, x_{N}\right\}$ be a minimizing $N$-point $s$-energy configuration on $\mathbb{S}^{d}$ and, for each $i=1, \ldots, N$, let $\delta_{i}:=\min _{j \neq i}\left|x_{i}-x_{j}\right|$. With $C\left(x_{i}, \delta_{i} / 2\right)$ denoting the spherical cap with center $x_{i}$ and Euclidean radius $\delta_{i} / 2$, we deduce that $\sum_{i=1}^{N} \mathcal{H}_{d}\left(C\left(x_{i}, \delta_{i} / 2\right)\right) \leq \mathcal{H}_{d}\left(\mathbb{S}^{d}\right)$. It is easily verified that

$$
\mathcal{H}_{d}\left(C\left(x_{i}, r\right)\right)=\mathcal{H}_{d}\left(S^{d}\right) \frac{r^{d}}{\lambda_{d} d}+\mathcal{O}\left(r^{d+2}\right), \quad r \rightarrow 0^{+},
$$

where

$$
\lambda_{d}:=\int_{-1}^{1}\left(1-t^{2}\right)^{\frac{d-2}{2}} d t=\frac{\sqrt{\pi} \Gamma\left(\frac{d}{2}\right)}{\Gamma\left(\frac{d+1}{2}\right)} .
$$

Thus for $1>\epsilon>0$ and all $N$ sufficiently large we have from the asymptotic denseness of the minimizing configurations (Theorem 1.1) that

$$
(1-\epsilon) \mathcal{H}_{d}\left(S^{d}\right) \frac{1}{2^{d} \lambda_{d} d} \sum_{i=1}^{N} \delta_{i}^{d} \leq \sum_{i=1}^{N} \mathcal{H}_{d}\left(C\left(x_{i}, \delta_{i} / 2\right)\right) \leq \mathcal{H}_{d}\left(\mathbb{S}^{d}\right)
$$

and so

$$
\sum_{i=1}^{N} \delta_{i}^{d} \leq(1-\epsilon)^{-1} 2^{d} \lambda_{d} d
$$

By convexity, we also have

$$
\mathcal{E}_{s}\left(\mathbb{S}^{d}, N\right)=\sum_{i \neq j} \frac{1}{\left|x_{i}-x_{j}\right|^{s}} \geq \sum_{i=1}^{N} \frac{1}{\delta_{i}^{s}}=\sum_{i=1}^{N}\left(\delta_{i}^{d}\right)^{-s / d} \geq N\left(\frac{1}{N} \sum_{i=1}^{N} \delta_{i}^{d}\right)^{-s / d} .
$$

Consequently, from (7) we obtain for $N$ large

$$
\frac{\mathcal{E}_{s}\left(\mathbb{S}^{d}, N\right)}{N^{1+s / d}} \geq\left((1-\epsilon)^{-1} 2^{d} \lambda_{d} d\right)^{-s / d}
$$

Letting first $N \rightarrow \infty$ and then $\epsilon \rightarrow 0$, Theorem 1.1 yields the estimate

$$
C_{s, d} \geq \Theta_{s, d}:=\left(\frac{\mathcal{H}_{d}\left(\mathbb{S}^{d}\right)}{2^{d} \lambda_{d} d}\right)^{s / d}=\frac{1}{2^{s}}\left(\mathcal{H}_{d-1}\left(\mathbb{S}^{d-1}\right) / d\right)^{s / d}
$$

A less trivial lower bound is the following, established in [6]:

Proposition 1.3. If $d \geq 2$ and $s>d$, then for $(s-d) / 2$ not an integer,

$$
C_{s, d} \geq \xi_{s, d}:=\left[\frac{\pi^{d / 2} \Gamma\left(1+\frac{s-d}{2}\right)}{\Gamma\left(1+\frac{s}{2}\right)}\right]^{s / d} \frac{d}{s-d} .
$$

Our main result for Riesz potentials is the following improvement over the lower bounds for $C_{s, d}$ in $(8)$ and Proposition 1.3

Theorem 1.4. For a fixed dimension $d$, let $z_{i}$ be the $i$-th smallest positive zero of the Bessel function $J_{d / 2}(z), i=1,2, \ldots$ Then, for $s>d$, 


$$
C_{s, d} \geq A_{s, d}
$$

where

$$
A_{s, d}:=\left[\frac{\pi^{\frac{d+1}{2}} \Gamma(d+1)}{\Gamma\left(\frac{d+1}{2}\right)}\right]^{s / d} \frac{4}{\lambda_{d} \Gamma(d+1)} \sum_{i=1}^{\infty}\left(z_{i}\right)^{d-s-2}\left(J_{d / 2+1}\left(z_{i}\right)\right)^{-2}
$$

and $\lambda_{d}$ is as defined in (6).

For $d=1, A_{s, d}=2 \zeta(s)$, which is optimal. Furthermore, as we prove in Section 3 , both $A_{s, d}$ and $\xi_{s, d}$ have the same dominant behavior as $C_{s, d}$ as $s \rightarrow$ $d^{+}$; namely they all have a simple pole at $s=d$ with the same residue. In Figure 1. we compare the bounds $A_{s, 2}, \Theta_{s, 2}, \xi_{s, 2}$ with the conjectured value $\widetilde{C}_{2, s}$.

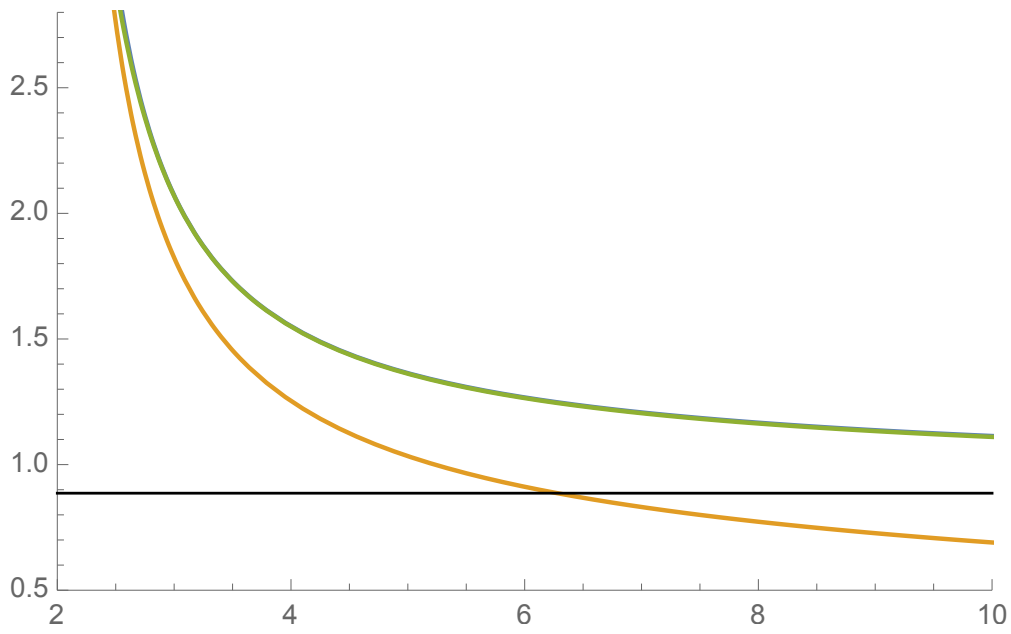

Figure 1: The two lower graphs show $\Theta_{s, 2}^{1 / s}$ (constant graph) and $\xi_{s, 2}^{1 / s}$ while the upper two graphs (indistinguishable on this scale) show both $A_{s, 2}^{1 / s}$ and the conjectured value $\widetilde{C}_{2, s}^{1 / s}$ as $s$ ranges from 2 to 10 .

Proposition 1.5. Let $d \in \mathbb{N}$. Then

$$
\lim _{s \rightarrow d^{+}}(s-d) C_{s, d}=\lim _{s \rightarrow d^{+}}(s-d) \xi_{s, d}=\lim _{s \rightarrow d^{+}}(s-d) A_{s, d}=\frac{2 \pi^{d / 2}}{\Gamma\left(\frac{d}{2}\right)} .
$$

As illustrated at the end of Section 2 , the Levenshtein $1 / N$-quadrature rules give bounds on the minimal separation distance for optimal packings on $\mathbb{S}^{d}$, and $A_{s, d}$ recovers these bounds as $s \rightarrow \infty$. For $d=2,4,8$, and 24 , letting $\widetilde{C}_{s, d}$ be the conjectured values of $C_{s, d}$ from Conjecture 1.2 , it is easy to verify that $\lim _{s \rightarrow \infty}\left(\widetilde{C}_{s, d} / A_{s, d}\right)^{1 / s}$ exists. Numerical comparisons between $A_{s, d}$ and $\widetilde{C}_{s, d}$ are illustrated in Section 4.

We next consider bounds for the Gaussian energy of infinite point configurations in $\mathbb{R}^{d}$. Our goal is to show that the method used to prove Theorem 
1.4 provides an alternative approach to deriving the lower bounds obtained by Cohn and de Courcy-Ireland [7. We begin with some essential definitions.

Definition 1.6. For an infinite configuration $\mathcal{C} \subset \mathbb{R}^{d}$ and $f:(0, \infty) \rightarrow \mathbb{R}$, the lower f-energy of $\mathcal{C}$ is

$$
E_{f}(\mathcal{C}):=\liminf _{R \rightarrow \infty} \frac{1}{\#\left(\mathcal{C} \cap B^{d}(R)\right)} \sum_{\substack{x, y \in \mathcal{C} \cap B^{d}(R) \\ x \neq y}} f(|x-y|),
$$

where \# denotes cardinality and $B^{d}(R)$ is the $d$-dimensional ball of radius $R$ centered at 0 . If the limit exists, we call it the f-energy of $\mathcal{C}$.

Definition 1.7. The lower density $\rho$ of a configuration $\mathcal{C}$ is defined to be

$$
\rho:=\liminf _{R \rightarrow \infty} \frac{\#\left(\mathcal{C} \cap B^{d}(R)\right)}{\operatorname{vol}\left(B^{d}(R)\right)} .
$$

If the limit exists, we call it the density of $\mathcal{C}$.

We shall show that universal lower bounds developed in 5 and based on Delsarte-Levenshtein methods can be used to prove the following estimate of Cohn and de Courcey-Ireland. (The results in [7] came to the authors' attention during the preparation of this manuscript and appear in the dissertation of Michaels 22.)

Theorem 1.8 ([7]). Let $f(|x-y|)=e^{-\alpha|x-y|^{2}}, \alpha>0$, be a Gaussian potential in $\mathbb{R}^{d}$ and choose $R_{\rho}$ so that $\operatorname{vol}\left(B^{d}\left(R_{\rho} / 2\right)\right)=\rho$. Then the minimal $f$-energy for point configurations of density $\rho$ in $\mathbb{R}^{d}$ is bounded below by

$$
\frac{4}{\lambda_{d} \Gamma(d+1)} \sum_{i=1}^{\infty} z_{i}^{d-2}\left(J_{d / 2+1}\left(z_{i}\right)\right)^{-2} f\left(\frac{z_{i}}{\pi R_{\rho}}\right),
$$

where the $z_{i}$ 's are as in Theorem 1.4 .

We remark that there is a strong relation connecting Theorems 1.8 and 1.4 Indeed, if $f(r)=g\left(r^{2}\right)$ for some completely monotone function $g$ with sufficient decay, then there is some non-negative measure $\mu$ on $[0, \infty)$ such that (e.g., see [28])

$$
f(r)=\int_{0}^{\infty} e^{-\alpha r^{2}} d \mu(\alpha) .
$$

Then it follows that Theorem 1.8 also holds for such $f$ and, in particular, for hypersingular Riesz $s$-potentials $f_{s}(r)=\left(r^{2}\right)^{-s / 2}$ for $s>d$. Furthermore, it is shown in 14 that the constant $C_{s, d}$ also appears in the context of minimizing the Riesz $s$-energy over infinite point configurations $\mathcal{C} \subset \mathbb{R}^{d}$ with a fixed density $\rho$ :

$$
C_{s, d}=\inf _{\substack{\mathcal{C} \text { has } \\ \text { density } 1}} E_{f_{s}}(\mathcal{C}) .
$$

Combining 13 and Theorem 1.8 then provides an alternate proof of Theorem 1.4

An outline of the remainder of this article is as follows. In Section 2, we describe the Delsarte-Yudin linear programming lower bounds and the Levenshtein $1 / N$-quadrature rules. More thorough treatments can be found in [1], 4, 
and [20]. In Section 3, we present the proofs of Theorem 1.4 Proposition 1.5 . and Theorem 1.8 using an asymptotic result on Jacobi polynomials from Szegó 24. Finally, in Section 4, we discuss numerically the quality of the bound $A_{s, d}$ and formulate a natural conjecture.

\section{Linear Programming Bounds}

For $\alpha, \beta>-1$, let $\left\{P_{k}^{(\alpha, \beta)}\right\}_{k=1}^{\infty}$ denote the sequence of Jacobi polynomials of respective degrees $k$ that are orthogonal with respect to the weight $\omega^{\alpha, \beta}(t):=$ $(1-t)^{\alpha}(1+t)^{\beta}$ on $[-1,1]$ and normalized by

$$
P_{k}^{(\alpha, \beta)}(1)=1
$$

While this normalization is crucial for the linear programming methods presented here, we note that many authors choose $P_{k}^{(\alpha, \beta)}(1)=\left(\begin{array}{c}k+\alpha \\ k\end{array}\right)$. For a fixed dimension $d \geq 1$, the Gegenbauer or ultraspherical polynomials are given by $P_{k}(t):=P_{k}^{\left(\frac{d-2}{2}, \frac{d-2}{2}\right)}(t)$ with weight $\omega_{d}(t):=\omega^{\left(\frac{d-2}{2}, \frac{d-2}{2}\right)}(t)$. For our purposes, the so-called adjacent polynomials

$$
P_{k}^{a, b}(t):=P_{k}^{\left(\frac{d-2}{2}+a, \frac{d-2}{2}+b\right)}(t), \quad a, b \in\{0,1\}
$$

associated with the weights $\omega_{d}^{a, b}(t):=(1-t)^{a}(1+t)^{b} \omega_{d}(t)$, play an essential role.

For functions $f:[-1,1] \rightarrow \mathbb{R}$ that are square integrable with respect to $\omega_{d}$ on $[-1,1]$, we consider its Gegenbauer expansion: $f(t)=\sum_{i=0}^{\infty} f_{k} P_{k}(t)$, where the $f_{k}$ 's are given by

$$
f_{k}:=\frac{\int_{-1}^{1} f(t) P_{k}(t) \omega_{d}(t) d t}{\int_{-1}^{1}\left[P_{k}(t)\right]^{2} \omega_{d}(t) d t} .
$$

The following result forms the basis for the linear programming bounds for packing and energy on the sphere (see, for example, [1] or [1, Theorem 5.3.2]):

Theorem 2.1. If $f:[-1,1] \rightarrow \mathbb{R}$ is of the form

$$
f(t)=\sum_{k=0}^{\infty} f_{k} P_{k}(t)
$$

with $f_{k} \geq 0$ for all $k \geq 1$ and $\sum_{k=0}^{\infty} f_{k}<\infty$, then for any $N$-point subset $\omega_{N}=\left\{x_{1}, \ldots, x_{N}\right\} \subset \mathbb{S}^{d}$,

$$
\sum_{1 \leq i \neq j \leq N} f\left(\left\langle x_{i}, x_{j}\right\rangle\right) \geq f_{0} N^{2}-f(1) N .
$$

Moreover, if $h:[-1,1] \rightarrow[0, \infty]$ and $h(t) \geq f(t)$ on $[-1,1]$, then for the energy kernel $K(x, y):=h(\langle x, y\rangle)$,

$$
E_{K}\left(\omega_{N}\right) \geq \mathcal{E}_{K}\left(\mathbb{S}^{d}, N\right) \geq f_{0} N^{2}-f(1) N .
$$

Equality holds in (18) and $\omega_{N}$ is an optimal (minimizing) h-energy configuration if and only if 
(i) $h(t)=f(t)$ for all $t \in\left\{\left\langle x_{i}, x_{j}\right\rangle: i \neq j\right\}$ and

(ii) for all $k \geq 1$, either $f_{k}=0$ or $\sum_{i, j=1}^{N} P_{k}\left(\left\langle x_{i}, x_{j}\right\rangle\right)=0$.

An $N$-point configuration $\omega_{N}=\left\{x_{i}\right\}_{i=1}^{N} \subset \mathbb{S}^{d}$ is called a spherical $\tau$-design if

$$
\int_{S^{d}} f(x) d \sigma_{d}(x)=\frac{1}{N} \sum_{i=1}^{N} f\left(x_{i}\right)
$$

holds for all spherical polynomials $f$ of degree at most $\tau$, where $\sigma_{d}$ denotes the normalized surface area measure on $\mathbb{S}^{d}$. Using Theorem 2.1. Delsarte, Goethals, and Seidel [12] obtained an estimate for the minimum number of points on $\mathbb{S}^{d}$ that are necessary for a $\tau$-design. Namely, setting

$$
B(d, \tau):=\min \left\{N: \exists \omega_{N} \subset \mathbb{S}^{d} \text { a spherical } \tau \text {-design }\right\},
$$

they show

$$
B(d, \tau) \geq D(d, \tau):= \begin{cases}2\left(\begin{array}{c}
d+k-1 \\
d
\end{array}\right) & \text { if } \tau=2 k-1, \\
\left(\begin{array}{c}
d+k \\
d
\end{array}\right)+\left(\begin{array}{c}
d+k-1 \\
d
\end{array}\right) & \text { if } \tau=2 k .\end{cases}
$$

Definition 2.2. A sequence of ordered pairs $\left\{\left(\alpha_{i}, \rho_{i}\right)\right\}_{i=1}^{k}$ is said to be a $1 / N$ quadrature rule exact on a subspace $\Lambda \subset C([-1,1])$ if $1>\alpha_{1}>\cdots>\alpha_{k} \geq-1$, $\rho_{i}>0$ for $i=1, \ldots, k$, and for all $f \in \Lambda$,

$$
f_{0}=\frac{1}{\lambda_{d}} \int_{-1}^{1} f(t) \omega_{d}(t) d t=\frac{f(1)}{N}+\sum_{i=1}^{k} \rho_{i} f\left(\alpha_{i}\right) .
$$

Theorem 2.1 gives rise immediately to the following:

Theorem 2.3. Let $\left\{\left(\alpha_{i}, \rho_{i}\right)\right\}_{i=1}^{k}$ be a $1 / N$-quadrature rule exact on a subspace $\Lambda$. For $K(x, y):=h(\langle x, y\rangle)$, let $\mathcal{A}_{h}$ be the set of functions $f$ with $f(t) \leq h(t)$ on $[-1,1]$ that satisfy the hypotheses of Theorem 2.1. Then

$$
\mathcal{E}_{K}\left(\mathbb{S}^{d}, N\right) \geq N^{2} \sum_{i=1}^{k} \rho_{i} f\left(\alpha_{i}\right)
$$

and

$$
\sup _{f \in \Lambda \cap \mathcal{A}_{h}} N^{2} \sum_{i=1}^{k} \rho_{i} f\left(\alpha_{i}\right) \leq N^{2} \sum_{i=1}^{k} \rho_{i} h\left(\alpha_{i}\right)
$$

Levenshtein derives a $1 / N$-quadrature given in Theorem 2.4 below to obtain the following bound for the maximal cardinality of a configuration $\omega_{N} \subset \mathbb{S}^{d}$ with largest inner product $s$. Let

$$
A(d, s):=\max \left\{N: \exists \omega_{N} \subset \mathbb{S}^{d} \text { with }\left\langle x_{i}, x_{j}\right\rangle \leq s, i \neq j\right\} .
$$


Letting $\gamma_{k}^{a, b}$ denote the greatest zero of $P_{k}^{a, b}$, we partition $[-1,1]$ into the following disjoint union of countably many intervals. For $\tau=1,2, \ldots$,

$$
I_{\tau}:= \begin{cases}{\left[\gamma_{k-1}^{1,1}, \gamma_{k}^{1,0}\right]} & \text { if } \tau=2 k-1 \\ {\left[\gamma_{k}^{1,0}, \gamma_{k}^{1,1}\right]} & \text { if } \tau=2 k\end{cases}
$$

which are well defined by the interlacing properties $\gamma_{k-1}^{1,1}<\gamma_{k}^{1,0}<\gamma_{k}^{1,1}$. Then

$$
A(d, s) \leq L(d, s)
$$

where

$$
L(d, s):= \begin{cases}L_{2 k-1}(d, s):=\left(\begin{array}{c}
k+d-2 \\
k-1
\end{array}\right)\left[\frac{2 k+d-2}{d}-\frac{P_{k-1}(s)-P_{k}(s)}{(1-s) P_{k}(s)}\right] & \text { if } s \in I_{2 k-1}, \\
L_{2 k}(d, s):=\left(\begin{array}{c}
k+d-1 \\
k
\end{array}\right)\left[\frac{2 k+d}{d}-\frac{(1+s)\left(P_{k}(s)-P_{k+1}(s)\right)}{(1-s)\left(P_{k}(s)+P_{k+1}(s)\right)}\right] & \text { if } s \in I_{2 k} .\end{cases}
$$

The function $L(d, s)$ is called the Levenshtein function. For fixed $d$, it is continuous and increasing in $s$ on $[-1,1]$. The formula for the Levenshtein function is such that the quadrature nodes given in Theorem 2.4 below will have weight $1 / N$ at the node $\alpha_{0}=1$. At the endpoints of the intervals $\mathcal{I}_{\tau}$,

$$
\begin{aligned}
& L_{2 k-2}\left(d, \gamma_{k-1}^{1,1}\right)=L_{2 k-1}\left(d, \gamma_{k-1}^{1,1}\right)=D(d, 2 k-1), \\
& L_{2 k-1}\left(d, \gamma_{k-1}^{1,0}\right)=L_{2 k}\left(d, \gamma_{k-1}^{1,0}\right)=D(d, 2 k),
\end{aligned}
$$

where $L_{\tau}$ denotes the restriction of $L$ to the interval $I_{\tau}$.

Setting

$$
r_{i}^{a, b}:=\left(\frac{1}{\lambda_{d}^{a, b}} \int_{-1}^{1}\left(P_{i}^{a, b}(t)\right)^{2} \omega_{d}^{a, b}(t) d t\right)^{-1},
$$

where $\lambda_{d}^{a, b}:=\int_{-1}^{1} \omega_{d}^{a, b}(t) d t$, we define

$$
Q_{k}^{a, b}(x, y):=\sum_{i=0}^{k} r_{i}^{a, b} P_{i}^{a, b}(x) P_{i}^{a, b}(y) .
$$

By the Christoffel Darboux formula (see [24, Section 3.2]),

$$
\begin{aligned}
& Q_{k}^{a, b}(x, y)=r_{k}^{a, b} m_{k}^{a, b}\left(\frac{P_{k+1}^{a, b}(x) P_{k}^{a, b}(y)-P_{k}^{a, b}(x) P_{k+1}^{a, b}(y)}{x-y}\right), \quad x \neq y \\
& Q_{k}^{a, b}(x, x)=r_{k}^{a, b} m_{k}^{a, b}\left[\left(P_{k+1}^{a, b}\right)^{\prime}(x) P_{k}^{a, b}(x)-\left(P_{k}^{a, b}\right)^{\prime}(x) P_{k+1}^{a, b}(x)\right],
\end{aligned}
$$

where $m_{i}^{a, b}:=l_{i}^{a, b} / l_{i+1}^{a, b}$ and $l_{i}^{a, b}$ is the leading coefficient of $P_{i}^{a, b}$.

The following $1 / N$-quadrature rule proven in [19, Theorems 4.1 and 4.2 ] plays an essential role in establishing Theorem 1.4

Theorem 2.4. For $N \in \mathbb{N}$, let $\tau$ be such that $N \in(D(d, \tau), D(d, \tau+1)]$, and let $\alpha_{1}=\beta_{1}=s$ be the unique solution to 


$$
N=L(d, s) .
$$

(i) If $\tau=2 k-1$, define nodes $1>\alpha_{1}>\cdots>\alpha_{k}>-1$ as the solutions of

$$
(t-s) Q_{k-1}^{1,0}(t, s)=0
$$

with associated weights

$$
\rho_{i}=\frac{\lambda_{d}^{1,0}}{\lambda_{d}\left(1-\alpha_{i}\right) Q_{k-1}^{1,0}\left(\alpha_{i}, \alpha_{i}\right)} .
$$

Then $\left\{\left(\alpha_{i}, \rho_{i}\right)\right\}_{i=1}^{k}$ is a $1 / N$-quadrature rule exact on $\Pi_{2 k-1}$.

(ii) If $\tau=2 k$, define nodes $1>\beta_{1}>\cdots>\beta_{k+1}=-1$ as the solutions of

$$
(1+t)(t-s) Q_{k-1}^{1,1}(t, s)=0
$$

with associated weights

$$
\begin{aligned}
\eta_{i} & =\frac{\lambda_{d}^{1,1}}{\left(1-\beta_{i}^{2}\right) Q_{k}^{1,1}\left(\beta_{i}, \beta_{i}\right)}, \quad i=1, \ldots, k, \\
\eta_{k+1} & =\frac{Q_{k}(s, 1)}{Q_{k}(-1,-1) Q_{k}(s, 1)-Q_{k}(-1,1) Q_{k}(s,-1)} .
\end{aligned}
$$

Then $\left\{\left(\beta_{i}, \eta_{i}\right)\right\}_{i=1}^{k+1}$ is a $1 / N$-quadrature rule exact on $\Pi_{2 k}$.

Here and below $\Pi_{m}$ denotes the collection of all algebraic polynomials of degree at most $m$.

Remark 2.5. At the endpoints we also have that for $N=D(d, 2 k),\left\{\left(\alpha_{i}, \rho_{i}\right)\right\}_{i=1}^{k}$ is exact on $\Pi_{2 k}$ and for $N=D(d, 2 k+1),\left\{\left(\beta_{i}, \eta_{i}\right)\right\}_{i=1}^{k+1}$ is exact on $\Pi_{2 k+1}$.

The above quadrature rules were used by Boyvalenkov et. al to derive the following universal lower bounds for the energy of spherical configurations.

Theorem 2.6. (5) Let $N$ be fixed and $h(t)$ denote an absolutely monotone potential on $[-1,1)$. Suppose $\tau=\tau(d, N)$ is such that $N \in(D(d, \tau), D(d, \tau+1)]$ and let $k=\left\lceil\frac{\tau+1}{2}\right\rceil$. If $\left\{\left(\alpha_{i}, \rho_{i}\right)\right\}_{i=1}^{k}$ is the $1 / N$-quadrature rule of Theorem 2.4 then

$$
\mathcal{E}_{h}\left(\mathbb{S}^{d}, N\right) \geq N^{2} \sum_{i=1}^{k} \rho_{i} h\left(\alpha_{i}\right)
$$

An analogous statement holds for the pairs $\left(\beta_{i}, \eta_{i}\right)$ of Theorem 2.4(ii), but we shall not make use of it in our proofs.

Taking into account Theorem 2.3 inequality 29 ) provides an optimal linear programming lower bound for the subspace $\Lambda=\Pi_{k}$. As an application, we now show that Theorem 2.6 recovers the first-order asymptotics for integrable potentials. 
Example 2.7. If $h(t)$ is any absolutely monotone function that is also integrable with respect to $\omega_{d}(t)$ on $[-1,1]$, then

$$
\lim _{N \rightarrow \infty} \frac{\mathcal{E}_{h}\left(\mathbb{S}^{d}, N\right)}{N^{2}} \geq \frac{1}{\lambda_{d}} \int_{-1}^{1} h(t) \omega_{d}(t) d t,
$$

where $\lambda_{d}$ is defined in (6).

Remark 2.8. It is a classical result of potential theory that the limit exists and equality holds in (30); see [17].

Proof of (30). First suppose $h(t)$ is continuous on $[-1,1]$. For $\epsilon>0$, let $f(t)$ be a polynomial of degree $\leq 2 k-1$ such that $|f(t)-h(t)| \leq \epsilon$ uniformly on $[-1,1]$. Setting $\left(\alpha_{0}, \rho_{0}\right):=(1,1 / N)$, we note that the weights $\rho_{i}$ given in 26 are positive for $i=0, \ldots, k$ and that $\sum_{i=0}^{k} \rho_{i}=1$. From 19 , we have with $\alpha_{i}=\alpha_{i}(N), \rho_{i}=\rho_{i}(N), k=k(N)$,

$$
\begin{aligned}
\mid \frac{1}{\lambda_{d}} \int_{-1}^{1} h(t) \omega_{d}(t) d t & -\sum_{i=0}^{k} \rho_{i} h\left(\alpha_{i}\right) \mid \\
& \leq \frac{1}{\lambda_{d}} \int_{-1}^{1}|h(t)-f(t)| \omega_{d}(t) d t+\sum_{i=0}^{k} \rho_{i}\left|f\left(\alpha_{i}\right)-h\left(\alpha_{i}\right)\right| \\
& \leq 2 \epsilon \rightarrow 0 \text { as } N \rightarrow \infty .
\end{aligned}
$$

Since $\rho_{0} h\left(\alpha_{0}\right)=h(1) / N \rightarrow 0$ as $N \rightarrow \infty$, inequality (30) follows.

Next suppose $h(t)$ is integrable and $g_{m} \nearrow h$ a sequence of continuous functions increasing to $h$ (for existence, consider $g_{m}(t):=h((1-1 / m)(t+1)-1)$ ). By the Monotone Convergence Theorem and a similar string of inequalities as above, it follows that

$$
\lim _{k \rightarrow \infty} \sum_{i=1}^{k} \rho_{i} h\left(\alpha_{i}\right)=\frac{1}{\lambda_{d}} \int_{-1}^{1} h(t) \omega_{d}(t),
$$

which concludes the proof.

We remark that another feature of Theorem 2.4 is that it includes a bestpacking result of Levenshtein [18, 20, which asserts the following: if $\omega_{N}=$ $\left\{x_{1}, \ldots, x_{N}\right\}$ is any $N$-point configuration on $\mathbb{S}^{d}$ and $\delta\left(\omega_{N}\right):=\max _{i \neq j}\left\langle x_{i}, x_{j}\right\rangle$, then

$$
\delta\left(\omega_{N}\right) \geq \alpha_{1},
$$

where $\alpha_{1}=\alpha_{1}(N)$ is as given in Theorem 2.4. This follows by considering absolutely monotone approximations to the potential

$$
h(t)= \begin{cases}\infty & \text { if } t \geq \alpha_{1} \\ 0 & \text { if } t<\alpha_{1}\end{cases}
$$

Indeed, if $\delta\left(\omega_{N}\right)<\alpha_{1}$, then $E_{h}\left(\omega_{N}\right)=0$, but $\sum_{i=1}^{k} \rho_{i} h\left(\alpha_{i}\right)=\infty$, contradicting 29 . 


\section{Proofs of Theorems 1.4, 1.8, and Proposition 1.5}

Our approach will be to find the asymptotic expansion of the right-hand side of (29) as $N \rightarrow \infty$. Throughout this section we assume that $\alpha, \beta>-1$. We will make use of the following result from Szegő (see [24, Theorem 8.1.1]) adjusted by normalization (14):

Theorem 3.1. Locally uniformly in the complex z-plane,

$$
\lim _{k \rightarrow \infty} P_{k}^{(\alpha, \beta)}\left(\cos \frac{z}{k}\right)=\lim _{k \rightarrow \infty} P_{k}^{(\alpha, \beta)}\left(1-\frac{z^{2}}{2 k^{2}}\right)=\Gamma(\alpha+1)\left(\frac{z}{2}\right)^{-\alpha} J_{\alpha}(z) .
$$

This gives the following immediate corollary:

Corollary 3.2. If $-1<\gamma_{k, k}<\cdots<\gamma_{k, 1}<1$ are the zeros of $P_{k}^{(\alpha, \beta)}$ and $z_{i}$ is the $i$-th smallest positive zero of the Bessel function $J_{\alpha}(z)$, then

$$
\lim _{k \rightarrow \infty} k \cos ^{-1}\left(\gamma_{k, i}\right)=z_{i}
$$

Recalling definition (15) and making use of well-known properties of the derivatives, norms, and leading coefficients of the Jacobi polynomials (see, e.g., [24, Chapter 4]) we obtain the following asymptotic formulas as $k \rightarrow \infty$ :

$$
\begin{aligned}
\frac{\mathrm{d}}{\mathrm{d} t} P_{k}^{1,0}(t) & =\frac{1}{2}(k+d) \frac{\left(\begin{array}{c}
k+\frac{d+2}{2} \\
k
\end{array}\right)}{\left(\begin{array}{c}
k+\frac{d}{2} \\
k
\end{array}\right)} P_{k-1}^{2,1}(t) \\
& =\left(\frac{k^{2}}{d+2}+o\left(k^{2}\right)\right) P_{k-1}^{2,1}(t) .
\end{aligned}
$$

Furthermore,

$$
\begin{aligned}
\frac{r_{k}^{1,0}}{\lambda_{d}^{1,0}} & =\left(\int_{-1}^{1}\left(P_{k}^{1,0}(t)\right)^{2} \omega^{1,0}(t) d t\right)^{-1} \\
& =\left(\frac{2^{d} \Gamma\left(k+\frac{d+2}{2}\right) \Gamma\left(k+\frac{d}{2}\right)}{\left(\begin{array}{c}
k+\frac{d}{2} \\
k
\end{array}\right)^{2}(2 k+d) \Gamma(k+d) \Gamma(k+1)}\right)^{-1} \\
& =\frac{k^{d+1}}{2^{d-1} \Gamma\left(\frac{d+2}{2}\right)^{2}}+o\left(k^{d+1}\right) .
\end{aligned}
$$

Lastly, recalling that $l_{k}^{1,0}$ is the leading coefficient of $P_{k}^{1,0}(t)$,

$$
l_{k}^{1,0}=\frac{\Gamma(2 k+d)}{\left(\begin{array}{c}
k+\frac{d}{2} \\
k
\end{array}\right) 2^{k} \Gamma(k+1) \Gamma(k+d)},
$$

which yields for the ratio

$$
\begin{aligned}
m_{k}^{1,0}=\frac{l_{k}^{1,0}}{l_{k+1}^{1,0}} & =\left(\frac{2(k+1)(k+d)}{(2 k+d+1)(2 k+d)}\right)\left(\frac{2 k+2+d}{2 k+2}\right) \\
& =\frac{1}{2}+o(1) .
\end{aligned}
$$


Remark 3.3. Generalizing equations 33 - 35 to $P_{k}^{(\alpha, \beta)}(t)$ we obtain

$$
\begin{aligned}
& \frac{\mathrm{d}}{\mathrm{d} t} P_{k}^{(\alpha, \beta)}(t)=\left(\frac{k^{2}}{2(\alpha+1)}+o\left(k^{2}\right)\right) P_{k-1}^{(\alpha+1, \beta+1)}(t), \\
& r_{k}^{(\alpha, \beta)}=O\left(k^{2 \alpha+1}\right), \text { and } \\
& m_{k}^{(\alpha, \beta)}=\frac{1}{2}+o(1) .
\end{aligned}
$$

We also need the following additional lemmas.

Lemma 3.4. Let $p_{k}(t):=P_{k}^{(\alpha, \beta)}(t)$ be a sequence of Jacobi polynomials. If $z \in \mathbb{R}$ is fixed such that $\lim _{k \rightarrow \infty} p_{k}\left(\cos \frac{z}{k}\right)=c$ and $\beta_{k},-1 \leq \beta_{k} \leq 1$, is a sequence satisfying

$$
\lim _{k \rightarrow \infty} k \cos ^{-1}\left(\beta_{k}\right)=z,
$$

then

$$
\lim _{k \rightarrow \infty} p_{k+j}\left(\beta_{k}\right)=c
$$

for any fixed $j \in \mathbb{Z}$.

Proof. First, since $\lim _{k \rightarrow \infty}(k+j) \cos ^{-1}\left(\beta_{k}\right)=\lim _{k \rightarrow \infty} k \cos ^{-1}\left(\beta_{k}\right)$, by making the substitution $k=k+j$ it suffices to establish equation 40 for the case $j=0$. From (39), we have that

$$
\epsilon_{k}:=\left|\beta_{k}-\cos \frac{z}{k}\right|=o\left(\frac{1}{k^{2}}\right)
$$

Applying the mean value theorem, equation $(36)$, and using the fact that $p_{k}$ is uniformly bounded in $k$ on $[-1,1]$ (see e.g. [13]) we get with $p_{k-1}^{1,1}:=P_{k-1}^{\alpha+1 \beta+1}$,

$$
\left|p_{k}\left(\beta_{k}\right)-p_{k}\left(\cos \frac{z}{k}\right)\right|=p_{k}^{\prime}\left(\xi_{k}\right) \epsilon_{k}=k^{2} \tilde{c} p_{k-1}^{1,1}\left(\xi_{k}\right) \epsilon_{k}=o(1)
$$

for some $\xi_{k}$ between $\beta_{k}$ and $\cos \left(\frac{z}{k}\right)$, and $\tilde{c}>0$.

A stronger version of Lemma 3.4 holds when $c=0$.

Lemma 3.5. Let $-1<\gamma_{k, k}<\cdots<\gamma_{k, 1}<1$ be the zeros of $p_{k}(t):=P_{k}^{(\alpha, \beta)}(t)$, and denote by $z_{i}$ the $i$-th smallest positive zero of the Bessel function $J_{\alpha}(z)$. Then for all $i=1,2, \ldots$,

$$
\lim _{k \rightarrow \infty} k p_{k-1}\left(\gamma_{k, i}\right)=2 \Gamma(\alpha+1)\left(\frac{z_{i}}{2}\right)^{-\alpha+1} J_{\alpha+1}\left(z_{i}\right) .
$$

Proof. By Corollary 3.2 .

$$
\gamma_{k, i}=1-\frac{z_{i}^{2}}{2 k^{2}}+o\left(\frac{1}{k^{2}}\right)
$$

which implies

$$
\delta_{k}:=\left|\gamma_{k, i}-\gamma_{k-1, i}\right|=\frac{z_{i}^{2}}{k^{3}}+o\left(\frac{1}{k^{3}}\right), \text { as } k \rightarrow \infty .
$$


By the interlacing properties of the zeros of Jacobi polynomials, we see that $\gamma_{k, i}>\gamma_{k-1, i}$ and we can drop the absolute value in $\delta_{k}$. Expanding the Taylor series for $p_{k-1}(t)$ around the zero $\gamma_{k-1, i}$, we have

$$
k p_{k-1}\left(\gamma_{k, i}\right)=k \delta_{k} p_{k-1}^{\prime}\left(\gamma_{k-1, i}\right)+\frac{k \delta_{k}^{2} p_{k-1}^{\prime \prime}\left(\gamma_{k-1, i}\right)}{2}+\cdots
$$

Each successive derivative term beyond the first has order $o(1)$ since by repeated application of 36 and Lemma $3.4 p_{k}^{(j)}(t)=O\left(k^{2 j}\right) p_{k-j}^{j, j}(t)=O\left(k^{2 j}\right)$ while on the other hand $\delta_{k}^{j}=O\left(1 / k^{3 j}\right)$. Thus,

$$
k p_{k-1}\left(\gamma_{k, i}\right)=\frac{z_{i}^{2}}{2(\alpha+1)} p_{k-2}^{1,1}\left(\gamma_{k-1, i}\right)+o(1) \text { as } k \rightarrow \infty .
$$

Now by Theorem 3.1 and Lemma 3.4, we obtain the result.

We are now ready to prove the main theorem.

Proof of Theorem 1.4. In the case of Riesz energy, we have

$$
K_{s}(x, y)=h_{s}(\langle x, y\rangle)=(2-2\langle x, y\rangle)^{-s / 2}
$$

We consider the subsequence

$$
N_{k}:=D(d, 2 k)=\left(\begin{array}{c}
d+k \\
d
\end{array}\right)+\left(\begin{array}{c}
d+k-1 \\
d
\end{array}\right)=\frac{2}{\Gamma(d+1)} k^{d}+o\left(k^{d}\right) .
$$

By Theorem 1.1 it suffices to prove

$$
\lim _{k \rightarrow \infty} \frac{\mathcal{E}_{s}\left(N_{k}, \mathbb{S}^{d}\right)}{N_{k}^{1+s / d}} \geq \frac{A_{s, d}}{\mathcal{H}_{d}\left(\mathbb{S}^{d}\right)^{s / d}}
$$

where

$$
\mathcal{H}_{d}\left(\mathbb{S}^{d}\right)=\frac{2 \pi^{\frac{d+1}{2}}}{\Gamma\left(\frac{d+1}{2}\right)} .
$$

Along the subsequence $N_{k}$, from $21, \alpha_{1}=\gamma_{k, 1}^{1,0}$, where $\gamma_{k, i}^{1,0}$ is the $i$-th largest zero of $P_{k}^{1,0}(t)$ and

$$
\begin{aligned}
\left(t-\alpha_{1}\right) Q_{k-1}^{1,0}\left(t, \alpha_{1}\right) & =r_{k-1} m_{k-1}\left(P_{k}^{1,0}(t) P_{k-1}^{1,0}\left(\alpha_{1}\right)-P_{k-1}^{1,0}(t) P_{k}^{1,0}\left(\alpha_{1}\right)\right) \\
& =r_{k-1} m_{k-1}\left(P_{k}^{1,0}(t) P_{k-1}^{1,0}\left(\alpha_{1}\right)\right)
\end{aligned}
$$

thus the quadrature nodes are given by

$$
\alpha_{i}=\gamma_{k, i}^{1,0}, \quad i=1,2, \ldots, k .
$$

For a fixed $m$ and all $k \geq m$ we have by Theorem 2.6

$$
\frac{\mathcal{E}_{s}\left(N_{k}\right)}{N_{k}^{1+s / d}} \geq \frac{\sum_{i=1}^{k} \rho_{i} h_{s}\left(\alpha_{i}\right)}{N_{k}^{-1+s / d}} \geq \frac{\sum_{i=1}^{m} \rho_{i} h_{s}\left(\alpha_{i}\right)}{N_{k}^{-1+s / d}}
$$


For a fixed $i \leq m$, we next establish asymptotics for $\rho_{i} h\left(\alpha_{i}\right)$. By Corollary 3.2 we have

$$
\lim _{k \rightarrow \infty} \frac{h_{s}\left(\alpha_{i}\right)}{k^{s}}=\lim _{k \rightarrow \infty} \frac{\left(2-2 \alpha_{i}\right)^{-s / 2}}{k^{s}}=\left(z_{i}\right)^{-s},
$$

and by 33 and Lemma 3.4

$$
\lim _{k \rightarrow \infty} \frac{\left(P_{k}^{1,0}\right)^{\prime}\left(\alpha_{i}\right)}{k^{2}}=\frac{\Gamma(d / 2+2)}{d+2}\left(\frac{z_{i}}{2}\right)^{-\frac{d+2}{2}} J_{d / 2+1}\left(z_{i}\right)
$$

Furthermore, from Lemma 3.5, it follows that

$$
\lim _{k \rightarrow \infty} k P_{k-1}^{1,0}\left(\alpha_{i}\right)=2 \Gamma(d / 2+1)\left(\frac{z_{i}}{2}\right)^{-\frac{d-2}{2}} J_{d / 2+1}\left(z_{i}\right) .
$$

From the weight formula given in equation 26 and the Cristoffel-Darboux formula (24) we deduce that

$$
\lim _{k \rightarrow \infty} k^{d} \rho_{i}=\lim _{k \rightarrow \infty} k^{d}\left(\frac{\lambda_{d}}{\lambda_{d}^{1,0}}\left(1-\alpha_{i}\right) r_{k-1}^{1,0} m_{k-1}^{1,0}\left(P_{k}^{1,0}\right)^{\prime}\left(\alpha_{i}\right) P_{k-1}^{1,0}\left(\alpha_{i}\right)\right)^{-1}
$$

and combining equations $(32),(34),(35),(44)$, and $(45)$, this yields

$$
\begin{aligned}
& \lim _{k \rightarrow \infty} k^{d} \rho_{i}= \\
& {\left[\lambda_{d}\left(\frac{z_{i}^{2}}{2}\right)\left(\frac{1}{2^{d-1} \Gamma(d / 2+1)^{2}}\right) \frac{1}{2}\left(\frac{\Gamma(d / 2+2)}{d+2}\left(\frac{z_{i}}{2}\right)^{-d / 2-1} J_{d / 2+1}\left(z_{i}\right)\right)\right.} \\
& \left.\cdot 2 \Gamma(d / 2+1)\left(\frac{z_{i}}{2}\right)^{-d / 2+1} J_{d / 2+1}\left(z_{i}\right)\right]^{-1}
\end{aligned}
$$

Simplifying gives

$$
\lim _{k \rightarrow \infty} k^{d} \rho_{i}=\frac{2}{\lambda_{d} z_{i}^{2-d}\left(J_{d / 2+1}\left(z_{i}\right)\right)^{2}} .
$$

Finally, combining the asymptotics for $N_{k}, h_{s}\left(\alpha_{i}\right)$, and $\rho_{i}$, equations (41), 43, , and 48) respectively, we obtain

$$
\lim _{k \rightarrow \infty} \frac{\rho_{i} h\left(\alpha_{i}\right)}{N_{k}^{s / d-1}}=\frac{2}{\lambda_{d}\left(\frac{2}{\Gamma(d+1)}\right)^{s / d-1} z_{i}^{2-d+s}\left(J_{d / 2+1}\left(z_{i}\right)\right)^{2}},
$$

and thus

$$
\frac{C_{s, d}}{\mathcal{H}_{d}\left(\mathbb{S}^{d}\right)^{s / d}}=\lim _{k \rightarrow \infty} \frac{\mathcal{E}_{s}\left(N_{k}, \mathbb{S}^{d}\right)}{N_{k}^{1+s / d}} \geq \sum_{i=1}^{m} \frac{2}{\lambda_{d}\left(\frac{2}{\Gamma(d+1)}\right)^{s / d-1} z_{i}^{2-d+s}\left(J_{d / 2+1}\left(z_{i}\right)\right)^{2}}
$$

Multiplying by $\mathcal{H}_{d}\left(\mathbb{S}^{d}\right)^{s / d}$ and letting $m \rightarrow \infty$ gives 42 and hence $(9)$. 
Proof of Proposition 1.5. We first establish the limit involving $\xi_{s, d}$ :

$$
\lim _{s \rightarrow d^{+}}(s-d) \xi_{s, d}=\lim _{s \rightarrow d^{+}} d\left[\frac{\pi^{d / 2} \Gamma\left(1+\frac{s-d}{2}\right)}{\Gamma\left(1+\frac{s}{2}\right)}\right]^{s / d}=\frac{d \pi^{d / 2}}{\Gamma\left(1+\frac{d}{2}\right)}=\frac{2 \pi^{d / 2}}{\Gamma\left(\frac{d}{2}\right)} .
$$

If $\Lambda$ is a $d$-dimensional lattice with co-volume $|\Lambda|>0$ then it is known (see [25]) that the Epstein zeta function has a simple pole at $s=d$ with residue

$$
\frac{2 \pi^{d / 2}}{\Gamma(d / 2)|\Lambda|} .
$$

Proposition 1.3 , the bound $(3)$, and $(50)$ then show

$$
\lim _{s \rightarrow d^{+}}(s-d) C_{s, d}=\frac{2 \pi^{d / 2}}{\Gamma\left(\frac{d}{2}\right)} .
$$

Finally, we establish the limit involving $A_{s, d}$. The well-known asymptotic behavior of $J_{\frac{d}{2}+1}(z)[24$, as $z \rightarrow \infty$, is given by

$$
J_{\frac{d}{2}+1}(z)=-\sqrt{\frac{2}{\pi z}}\left(\cos \left(z-(d-3) \frac{\pi}{4}\right)+O\left(z^{-3 / 2}\right)\right)
$$

and $z_{n}$, the $n$-th zero of the $J_{\frac{d}{2}}(z)$, is given by (see [27])

$$
z_{n}=n \pi+(d-1) \frac{\pi}{4}+O\left(n^{-1}\right) .
$$

Thus,

$$
J_{\frac{d}{2}+1}\left(z_{n}\right)^{-2}=\frac{\pi z_{n}}{2}+O\left(n^{-1}\right)
$$

and so we have

$$
\sum_{n=1}^{\infty} \frac{1}{z_{n}^{s-d+1} J_{\frac{d}{2}}\left(z_{n}\right)^{2}}=\frac{\pi}{2} \sum_{n=1}^{\infty} \frac{1}{z_{n}^{s-1}+a_{n}}=\frac{1}{2 \pi^{s-d}} \sum_{n=1}^{\infty} \frac{1}{\left(n+(d-1) / 4+b_{n}\right)^{s-1}+a_{n}}
$$

where $a_{n}, b_{n}=o(1)$. As $s \rightarrow d^{+}$, this sum approaches the Hurwitz zeta function, $\zeta(s-d+1,(d+3) / 4)$, where

$$
\zeta(s, q):=\sum_{n=0}^{\infty} \frac{1}{(n+q)^{s}} .
$$

That is,

$$
\lim _{s \rightarrow d^{+}} \frac{\sum_{n=1}^{\infty}\left(\left(n+(d-1) / 4+b_{n}\right)^{s-d+1}+a_{n}\right)^{-1}}{\zeta(s-d+1,(d+3) / 4)}=1 .
$$

Indeed, suppose $a=\sup \left|a_{n}\right|$ and $b=\sup \left|b_{n}\right|$. Then,

$$
\begin{aligned}
& \sum_{n=1}^{\infty} \frac{1}{\left(n+(d-1) / 4+b_{n}\right)^{s-d+1}+a_{n}} \geq \sum_{n=1}^{\infty} \frac{1}{(n+(d-1) / 4+b)^{s-d+1}+a} \\
& \geq \sum_{n=1}^{\infty} \frac{1}{(n+(d-1) / 4+b+a)^{s-d+1}}=\sum_{n=0}^{\infty} \frac{1}{\left(n+(d+3) / 4+b_{n}\right)^{s-d+1}+a_{n}} \\
& =\zeta(s-d+1,(d+3) / 4+a+b),
\end{aligned}
$$


and similarly

$$
\sum_{n=1}^{\infty} \frac{1}{\left(n+(d-1) / 4+b_{n}\right)^{s-d+1}+a_{n}} \leq \zeta(s-d+1,(d+3) / 4-a-b) .
$$

Since $\zeta(s, q) \rightarrow \infty$ as $s \rightarrow 1^{+}$(and the terms in the series in (56) stay bounded) the limit (56) holds. In fact $\zeta(s, q)$ has a simple pole of residue 1 at $s=1$ for all $q$ and so we obtain:

$$
\begin{aligned}
\lim _{s \rightarrow d^{+}}(s-d) A_{s, d} & =\lim _{s \rightarrow d^{+}}\left[\frac{\pi^{\frac{d+1}{2}} \Gamma(d+1)}{\Gamma\left(\frac{d+1}{2}\right)}\right]^{s / d} \frac{4(s-d)}{\lambda_{d} \Gamma(d+1)} \sum_{i=1}^{\infty}\left(z_{i}\right)^{d-s-2}\left(J_{d / 2+1}\left(z_{i}\right)\right)^{-2} \\
& =\lim _{s \rightarrow d^{+}} \frac{4 \pi^{d / 2}}{\Gamma\left(\frac{d}{2}\right)} \frac{(s-d)}{2} \zeta(s-d+1,(d+3) / 4)=\frac{2 \pi^{d / 2}}{\Gamma\left(\frac{d}{2}\right)}
\end{aligned}
$$

which completes the proof of Proposition 1.5 .

Proof of Theorem 1.8. For a fixed $\rho$ and a Gaussian potential $f(|x-y|)=$ $h(\langle x, y\rangle)=e^{-\alpha(2-2\langle x, y\rangle)}$, set

$$
c:=\left(a_{d} \rho\right)^{1 / d}, \text { where } a_{d}:=\frac{(d+1) \pi^{\frac{d+1}{2}}}{\Gamma\left(1+\frac{d+1}{2}\right)}=\frac{2 \pi^{\frac{d+1}{2}}}{\Gamma\left(\frac{d+1}{2}\right)}
$$

is the area of $\mathbb{S}^{d}$, and let

$$
f_{N}(|x-y|)=h_{N}(\langle x, y\rangle):=e^{-\alpha \frac{2-2\langle x, y\rangle}{\left(c N^{-1 / d}\right)^{2}}} .
$$

Our approach is to first obtain estimates for the $h_{N}$-energy of $N$-point configurations on the sphere $\mathbb{S}^{d}$.

For each $N, h_{N}$ is absolutely monotone on $[-1,1)$, and so Theorem 2.6 holds. We apply the same asymptotic argument as in the proof of Theorem 1.4 to $h_{N}(t)$. In particular we sample along the subsequence

$$
N_{k}:=D(d, 2 k),
$$

where the nodes $\alpha_{i}$ are given by the zeros of $P_{k}^{1,0}(t)$. Using the asymptotic formulas for $N_{k}$, the quadrature nodes $\alpha_{i}$, and the weights $\rho_{i}$, we obtain from Corollary 3.2 and 48 that

$$
\liminf _{N \rightarrow \infty} \frac{\mathcal{E}_{h_{N}}\left(\mathbb{S}^{d}, N\right)}{N} \geq \frac{4}{\lambda_{d} \Gamma(d+1)} \sum_{i=1}^{\infty} \frac{z_{i}^{d-2}}{\left(J_{d / 2+1}\left(z_{i}\right)\right)^{2}} e^{-\alpha\left(\frac{z_{i}}{c(2 / \Gamma(d+1))^{-1 / d}}\right)^{2}} .
$$

Let $0<\epsilon<1$. Then there is a collection $\left\{C\left(a_{\ell}, r_{\ell}\right): \ell=1,2, \ldots, L\right\}$ of disjoint closed spherical caps on $\mathbb{S}^{d}$ such that $r_{\ell}<\epsilon$ and

$$
\sum_{\ell=1}^{L} \mathcal{H}_{d}\left(C\left(a_{\ell}, r_{\ell}\right)\right) \geq(1-\epsilon) \mathcal{H}_{d}\left(\mathbb{S}^{d}\right)
$$

Using (5) and the fact that the caps are disjoint, it follows that there is a constant $\kappa_{1}>0$, independent of $\epsilon$, such that

$$
\left(1+\kappa_{1} \epsilon\right)^{-1} d \lambda_{d} \leq \sum_{\ell=1}^{L} r_{\ell}^{d} \leq d \lambda_{d}\left(1+\kappa_{1} \epsilon\right) .
$$


Furthermore, there are mappings $\phi_{\ell}: B^{d}\left(r_{\ell}\right) \rightarrow C\left(a_{\ell}, r_{\ell}\right), \ell=1,2, \ldots, L$ and a constant $\kappa_{2}$ (again independent of $\epsilon$ ) such that

$$
\left|\phi_{\ell}(x)-\phi_{\ell}(y)\right| \geq\left(1-\kappa_{2} \epsilon\right)|x-y|, \quad\left(x, y \in B^{d}\left(r_{\ell}\right)\right) .
$$

Let $\mathcal{C}$ be a configuration in $\mathbb{R}^{d}$ with density $\rho$ and $f_{\alpha}$-energy $E_{f_{\alpha}}(\mathcal{C})$; i.e., the limits in Definitions 1.6 and 1.7 both exist. Then, as $R \rightarrow \infty$, we have for any $\alpha>0$,

$$
\#\left(\mathcal{C} \cap B^{d}(R)\right)=\rho \operatorname{vol}\left(B^{d}(R)\right)(1+o(1)),
$$

and

$$
E_{f_{\alpha}}\left(\mathcal{C} \cap B^{d}(R)\right) \leq\left[\rho \operatorname{vol}\left(B^{d}(R)\right)\right] E_{f_{\alpha}}(\mathcal{C})(1+o(1)) .
$$

For $\ell=1,2, \ldots, L$, let

$$
\omega_{N}^{\ell}:=\phi_{\ell}\left(c N^{-1 / d} \mathcal{C} \cap B^{d}\left(r_{\ell} N^{1 / d} / c\right)\right),
$$

and

$$
\omega_{N}^{\mathcal{C}}:=\bigcup_{\ell=1}^{L} \omega_{N}^{\ell} .
$$

Observing that $\rho \operatorname{vol}\left(B^{d}(1)\right) d \lambda_{d} / c^{d}=1$, we see from (58) and (60) that as $N \rightarrow \infty$ the cardinality of $\omega_{N}^{\mathcal{C}}$ satisfies:

$$
\# \omega_{N}^{\mathcal{C}}=\sum_{\ell=1}^{L} \#\left(\mathcal{C} \cap B^{d}\left(r_{\ell} N^{1 / d} / c\right)\right) \geq\left(1+\kappa_{1} \epsilon\right)^{-1} N(1+o(1)) .
$$

Let $\delta$ denote the smallest distance between any pair of distinct spherical caps $C\left(a_{\ell}, r_{\ell}\right)$ and $C\left(a_{\ell^{\prime}}, r_{\ell^{\prime}}\right)$. The cross energy for $\ell \neq \ell^{\prime}$ satisfies

$$
\frac{E_{h_{N}}\left(\omega_{N}^{\ell}, \omega_{N}^{\ell^{\prime}}\right)}{N}:=\frac{1}{N} \sum_{\substack{x \in \omega_{N}^{\ell} \\ y \in \omega_{N}^{\ell^{\prime}}}} h_{N}(\langle x, y\rangle) \leq N \exp \left(-\frac{\alpha \delta^{2}}{c^{2}} N^{2 / d}\right)=o(1),
$$

as $N \rightarrow \infty$.

Using (59) and defining $\alpha_{\epsilon}=\alpha\left(1-\kappa_{2} \epsilon\right)^{2}$, we obtain

$$
\begin{aligned}
E_{h_{N}}\left(\omega_{N}^{\ell}\right) & =\sum_{\substack{x, y \in \mathcal{C} \cap B^{d}\left(r_{\ell} N^{1 / d} / c\right) \\
x \neq y}} \exp \left(-\alpha \frac{\left|\phi_{\ell}\left(c N^{-1 / d} x\right)-\phi_{\ell}\left(c N^{-1 / d} y\right)\right|^{2}}{\left(c N^{-1 / d}\right)^{2}}\right) \\
& \leq \sum_{\substack{x, y \in \mathcal{C} \cap B^{d}\left(r_{\ell} N^{1 / d} / c\right) \\
x \neq y}} \exp \left(-\alpha\left(1-\kappa_{2} \epsilon\right)^{2}|x-y|^{2}\right)=E_{f_{\alpha_{\epsilon}}}\left(\mathcal{C} \cap B^{d}\left(r_{\ell} N^{1 / d} / c\right)\right) \\
& \leq\left[\rho \operatorname{vol}\left(B^{d}(1)\right)\right]\left(r_{\ell}^{d} N / c^{d}\right) E_{f_{\alpha_{\epsilon}}}(\mathcal{C})(1+o(1))=\frac{N r_{\ell}^{d}}{d \lambda_{d}} E_{f_{\alpha_{\epsilon}}}(\mathcal{C})(1+o(1)) .
\end{aligned}
$$

Using the above estimate for $E_{h_{N}}\left(\omega_{N}^{\ell}\right)$ together with 63 and 64 we obtain as $N \rightarrow \infty$,

$$
\begin{aligned}
\frac{\mathcal{E}_{h_{N}}\left(\mathbb{S}^{d}, \# \omega_{N}^{\mathcal{C}}\right)}{\# \omega_{N}^{\mathcal{C}}} & \leq \frac{E_{h_{N}}\left(\omega_{N}^{\mathcal{C}}\right)}{\# \omega_{N}^{\mathcal{C}}} \leq\left(1+\kappa_{1} \epsilon\right) \sum_{\ell=1}^{L} \frac{E_{h_{N}}\left(\omega_{N}^{\ell}\right)}{N}(1+o(1))+o(1) \\
& \leq\left(1+\kappa_{1} \epsilon\right) \frac{1}{d \lambda_{d}}\left(\sum_{\ell=1}^{L} r_{\ell}^{d}\right) E_{f_{\alpha_{\epsilon}}}(\mathcal{C})(1+o(1))+o(1) \\
& \leq\left(1+\kappa_{1} \epsilon\right)^{2} E_{f_{\alpha_{\epsilon}}}(\mathcal{C})(1+o(1))+o(1),
\end{aligned}
$$


Taking the limit inferior as $N \rightarrow \infty$ and then $\epsilon \rightarrow 0$ in (65) and using (57) completes the proof.

\section{$4 \quad$ Numerics}

Translated into packing density and using Corollary 3.2 , inequality (31) provides an alternate proof of the following best-packing bound of Levenshtein [18]:

Corollary 4.1.

$$
\Delta_{d} \leq \frac{z_{1}^{d}}{\Gamma(d / 2+1)^{2} 4^{d}}=: L_{d}
$$

As $s \rightarrow \infty$, the series in $A_{s, d}$ is dominated by the first term $z_{1}^{-s}$ and using the asymptotics of $C_{s, d}$ in $(2)$, we see that

$$
\lim _{s \rightarrow \infty}\left[\frac{C_{s, d}}{A_{s, d}}\right]^{1 / s}=\left[\frac{L_{d}}{\Delta_{d}}\right]^{1 / d}=: B_{d} \geq 1
$$

The following table shows the values of $B_{d}$ in dimensions $d=1,2,3,8$, and 24 where $\Delta_{d}$ is known precisely. For $d=4,5,6,7$ where $\Delta_{d}$ is conjectured to be given by lattice packings, the table provides an upper bound for $B_{d}$.

Table 1: Upper Bounds on $B_{d}$

\begin{tabular}{|c|c|}
\hline$d$ & $B_{d}$ \\
\hline 1 & 1 \\
2 & 1.00589479 \\
3 & 1.02703993 \\
4 & 1.02440844 \\
5 & 1.03861371 \\
6 & 1.03461793 \\
7 & 1.03156355 \\
8 & 1.01742074 \\
24 & 1.02403055 \\
\hline
\end{tabular}

For $d=2,4,8$, and 24, where $\widetilde{C}_{s, d}$ is given in Conjecture 1.2 we plot

$$
f(s):=\left[\frac{\widetilde{C}_{s, d}}{A_{s, d}}\right]^{1 / s} .
$$

The Epstein zeta functions for the $D_{4}, E_{8}$, and Leech lattices are calculated using known formulas for the theta functions (see [10, Ch. 4])

$$
\Theta_{\Lambda}(z)=\sum_{x \in \Lambda} e^{i \pi z|x|^{2}}, \quad \operatorname{Im} z>0 .
$$

Since these three lattices have vectors whose squared norms are even integers, we let $q=e^{i \pi z}$ and write

$$
\Theta_{\Lambda_{d}}(z)=\sum_{m=1}^{\infty} N_{d}(m) q^{2 m}
$$



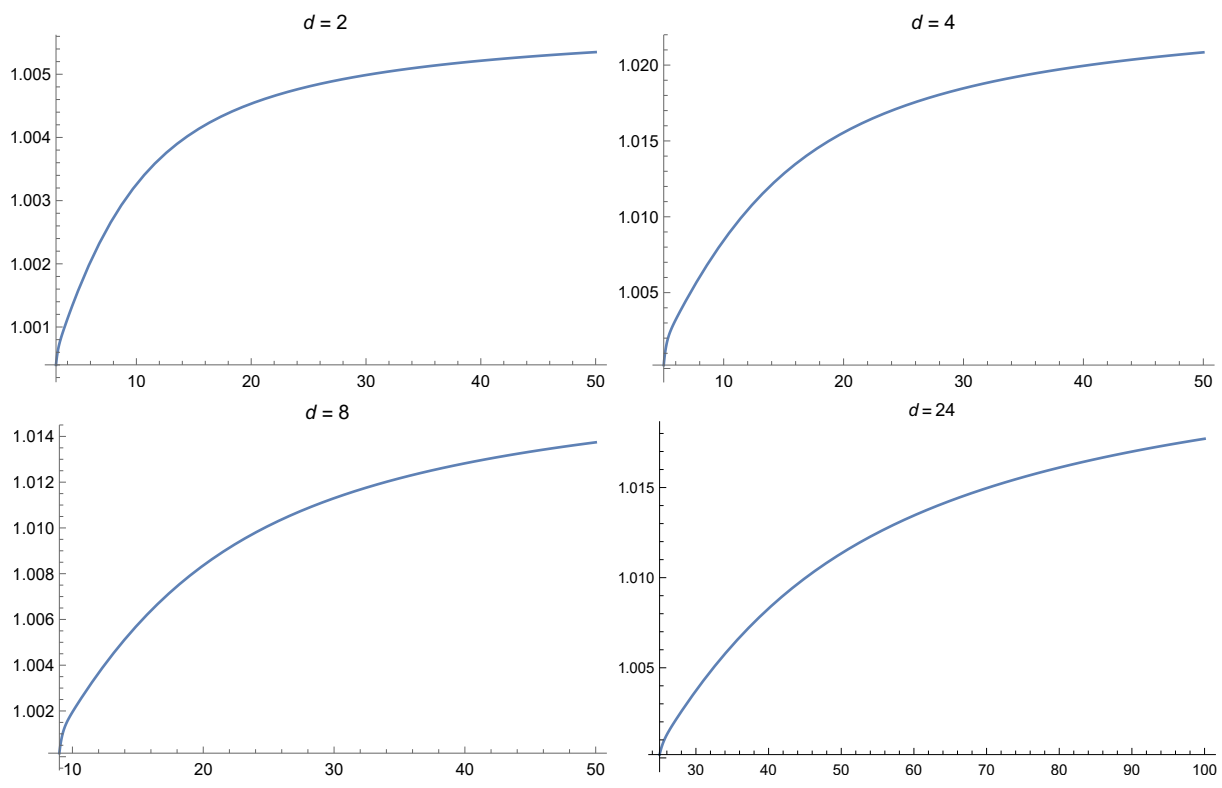

Figure 2: Graphs of $f(s)=\left(\widetilde{C}_{s, d} / A_{s, d}\right)^{1 / s}$ for $d=2,4,8$ and 24 .

where $N_{d}(m)$ counts the number of vectors in $\Lambda_{d}, d=4,8,24$ of squared norm $2 m$. Thus the Epstein zeta function

$$
\zeta_{\Lambda_{d}}(s)=\sum_{m=1}^{\infty} \frac{N_{d}(m)}{(2 m)^{s / 2}} .
$$

For the $D_{4}$ lattice, a classical result from number theory gives

$$
N_{4}(m)=24 \sum_{\substack{d \mid 2 m \\ d \text { odd }}} d .
$$

For the $E_{8}$ lattice, we have

$$
N_{8}(m)=240 \sigma_{3}(m)
$$

where

$$
\sigma_{k}(m)=\sum_{d \mid m} d^{k}
$$

is the divisor function. Finally for the Leech lattice, it is known that

$$
N_{24}(m)=\frac{65520}{691}\left(\sigma_{11}(m)-\tau(m)\right)
$$

where $\tau(m)$ is the Ramanujan tau function defined in [23].

Figure 2 plots $f(s)$ for $d=2,4,8$ and 24. In these dimensions the graphs monotonically increase to the limit $B_{d}$ as $s \rightarrow \infty$ and decrease to 1 as $s \rightarrow d^{+}$, demonstrating Proposition 1.5 . 
We remark that in high dimensions, it is likely that lattice packings are no longer optimal and less is known or conjectured regarding $C_{s, d}$. The Levenshtein packing bound from Corollary 4.1 yields for large $d$,

$$
\Delta_{d} \leq 2^{-0.5573 d}
$$

and thus

$$
B_{d}=O\left(\frac{2^{-0.5573}}{\Delta_{d}^{1 / d}}\right)
$$

Acknowledgment. The authors are grateful to J. S. Brauchart for his helpful suggestions.

\section{References}

[1] S. Borodachov, D. P. Hardin, and E. B. Saff. Minimal Discrete Energy on Rectifiable Sets. Springer, to appear.

[2] S. V. Borodachov, D. P. Hardin, and E. B. Saff. Asymptotics of bestpacking on rectifiable sets. Proc. Amer. Math. Soc., 135(8):2369-2380, 2007.

[3] S. V. Borodachov, D. P. Hardin, and E. B. Saff. Asymptotics for discrete weighted minimal Riesz energy problems on rectifiable sets. Trans. Amer. Math. Soc., 360(3):1559-1580, 2008.

[4] S. Boumova. Applications of Polynomials to Spherical Codes and Designs. $\mathrm{PhD}$ thesis, Eindhoven University of Technology, 2002.

[5] P. Boyvalenkov, P. Dragnev, D. P. Hardin, E. B. Saff, and M. Stoyanova. Universal lower bounds for potential energy of spherical codes. Constr. Approx., 44(3):385-415, 2016.

[6] J. S. Brauchart, D. P. Hardin, and E. B. Saff. The next-order term for optimal Riesz and logarithmic energy asymptotics on the sphere. In Recent Advances in Orthogonal Polynomials, Special Functions, and their Applications, volume 578 of Contemp. Math., pages 31-61. Amer. Math. Soc., Providence, RI, 2012.

[7] H. Cohn and M. de Courcy-Ireland. The Gaussian core model in high dimensions. Preprint, ArXiv: 1603.09684.

[8] H. Cohn and A. Kumar. Universally optimal distribution of points on spheres. J. Amer. Math. Soc., 10:99-148, 2006.

[9] H. Cohn, A. Kumar, S. Miller, D. Radchecnko, and M. Viazovska. The sphere packing problem in dimension 24. Preprint, arXiv:1603.06518.

[10] J. H. Conway and N. J. A. Sloane. Sphere Packings, Lattices and Groups, volume 290 of Grundlehren der Mathematischen Wissenschaften [Fundamental Principles of Mathematical Sciences]. Springer-Verlag, New York, third edition, 1999. 
[11] P. Delsarte. Bounds for unrestricted codes, by linear programming. Philips Res. Rep., 27:272-289, 1972.

[12] P. Delsarte, J. M. Goethals, and J. J. Seidel. Spherical codes and designs. Geometriae Dedicata, 6(3):363-388, 1977.

[13] T. Erdelyi, A. P. Magnus, and P. Nevai. Generalized Jacobi weights, Christoffel functions, and Jacobi polynomials. SIAM J. Math. Anal., 25:602-614, 1994.

[14] D. P. Hardin, T. Leblé, E. B. Saff, and S. Serfaty. Large deviation principles for hypersingular Riesz gases. Constr. Approx., to appear.

[15] D.P. Hardin and E.B. Saff. Minimal Riesz energy point configurations for rectifiable $d$-dimensional manifolds. Adv. Math., 193(1):174-204, 2005.

[16] A. B. J. Kuijlaars and E. B. Saff. Asymptotics for minimal discrete energy on the sphere. Trans. Amer. Math. Soc., 350(2):523-538, 1998.

[17] N.S. Landkof. Foundations of Modern Potential Theory, volume 180 of Grundlehren der Mathematischen Wissenschaften [Fundamental Principles of Mathematical Sciences]. Springer-Verlag, New York, 1972.

[18] V. I. Levenshtein. Bounds for packings in $n$-dimensional Euclidean space. Soviet Math. Dokl., 20:417-421, 1979.

[19] V. I. Levenshtein. Designs as maximum codes in polynomial metric spaces. Acta Appl. Math., 29(1-2):1-82, 1992.

[20] V. I. Levenshtein. Universal bounds for codes and designs. Chapter 6 in Handbook of Coding Theory, Eds. V. Pless and W.C. Huffman, Elsevier Science B.V., pages 499-648, 1998.

[21] A. Martinez-Finkelshtein, V. Maymeskul, E. A. Rakhmanov, and E. B. Saff. Asymptotics for minimal discrete Riesz energy on curves in $\mathbb{R}^{d}$. Canad. J. Math., 56(3):529-552, 2004.

[22] T. J. Michaels. Node Generation on Surfaces and Bounds on Minimal Riesz Energy. Ph.D. Thesis. Vanderbilt University, Nashville, TN, 2017.

[23] S. Ramanujan. On certain arithmetical functions [Trans. Cambridge Philos. Soc. 22 (1916), no. 9, 159-184]. In Collected papers of Srinivasa Ramanujan, pages 136-162. AMS Chelsea Publ., Providence, RI, 2000.

[24] G. Szegő. Orthogonal Polynomials. American Mathematical Society, Providence, R.I., fourth edition, 1975. American Mathematical Society, Colloquium Publications, Vol. XXIII.

[25] A. Terras. Harmonic analysis on symmetric spaces and applications. Number v. 1 in Harmonic Analysis on Symmetric Spaces and Applications. Springer-Verlag, 1988.

[26] M. Viazovska. The sphere packing problem in dimension 8. Preprint, arXiv:1603.04246. 
[27] G. N. Watson. A Treatise on the Theory of Bessel Functions. Cambridge Mathematical Library. Cambridge University Press, Cambridge, 1995. Reprint of the second (1944) edition.

[28] David Vernon Widder. The Laplace Transform. Princeton Mathematical Series, v. 6. Princeton University Press, Princeton, N. J., 1941.

[29] V. A. Yudin. Minimum potential energy of a point system of charges. Diskret. Mat., 4(2):115-121, 1992. 